Revista Iberoamericana, Vol. LXIX, Núm. 203, Abril-Junio 2003, 449-464

\title{
LOS ESTUDIOS CULTURALES EN LA ENCRUCIJADA DE LA INCERTIDUMBRE
}

\author{
POR \\ GEORGE YÚDICE \\ New York University
}

\section{INTRODUCCIÓN}

Dos hechos signan esta reflexión sobre los estudios culturales. En primer lugar, la declaración en el encuentro de LASA, en septiembre de 2001, de que se había terminado la alianza de los que venimos participando de los estudios culturales latinoamericanos (ECLA) en los últimos doce o trece años. En segundo lugar, el atentado a las torres gemelas pocos días después en New York y lo que significa este acontecimiento para el lugar que le atribuimos a la cultura, la identidad, la religión y, desde luego, la geopolítica. Ambos hechos constituyeron para mí un llamado a pensar autrement, como gustaba decir Foucault. Esta afirmación no quiere decir que vaya a acertar en la configuración de una nueva orientación analítica y crítica; más bien se trata de aprovechar mi estado de consternación para hacer un balance, o mejor un desordenamiento epistémico. Estamos acostumbrados a pensar que la cultura es un terreno importante de lucha social. Habría que preguntarse si el atentado no reorienta los estudios culturales hacia otros tipos de lucha. Debemos preguntarnos si en la nueva encrucijada es la cultura la que orientará hacia nuevas soluciones políticas y sociales - la esperanza de los que apostaron por el aporte de la sociedad civil en la época posdictatorial— o si emerge una nueva situación epocal en la que la noción misma y los usos de la cultura cambiarán.

\section{2. ¿Fin de la alianza de los estudios CUlturales latinoamericanos?}

En el tercer panel de la serie sobre los ECLA en LASA, de donde provienen muchos de los trabajos en este número de Revista Iberoamericana, Néstor García Canclini, tras escuchar las diatribas de John Beverley y otras declaraciones sobre la contradictoria institucionalización de los ECLA en las universidades estadounidenses y su inefectividad política, opinó que ya no existían las condiciones que gestaron un proyecto común. Acaso había llegado el momento de que cada uno siguiera su propio camino. Supongo que García Canclini hizo esta declaración para provocarnos a repensar y no necesariamente para que pongamos fin a las colaboraciones y los debates que han sido realmente enriquecedores.

Como no es mi propósito hacer una historia de los ECLA, apenas mencionaré algunos nombres para dar una idea de la variedad de acercamientos: mediáticos (Martín Barbero, 
Brunner, Landi), sobre culturas populares (García Canclini, Bonfil Batalla), sociológicos (Ortiz, Miceli, Lechner), antropológicos (Lins Ribeiro, Mato), marxistas (Dussel, Quijano), intelectuales y literarios (Rama, Ramos, Sarlo, Santiago, Schwarz), estéticos (Richard), filosóficos (Castro-Gómez), feministas (Lamas, Costa). Ya existen bastantes estudios que perfilan cánones y contra-cánones de los ECLA: Yúdice, Franco y Flores (1992), Beverley y Oviedo (1993), Castro-Gómez, González Stephan, Reynoso, Moraña, Mato y lo que ha aparecido en la Latin American Literary Review (1992) y en la Journal of Latin American Cultural Studies. Tampoco quisiera entrar en el debate sobre si lo que por conveniencia he llamado ECLA debiera llamarse crítica cultural, siguiendo a Nelly Richard, o estudios en cultura y poder, siguiendo a Mato.

Lo que sí quisiera destacar es el contraste entre EE.UU. y los países de América Latina en lo que respecta a la institucionalización de los ECLA. Estos han sido acogidos en EE.UU. en los departamentos de Letras o en programas de Comunicaciones, "Estudios de área” y "Estudios étnicos”, ${ }^{1}$ mientras que carecen casi totalmente de hogar académico en América Latina. Fuera del programa de García Canclini en la UAM-Iztapalapa, que se dedica a los Estudios sobre la Cultura Urbana, o el Programa Avançado de Cultura Contemporânea de Heloísa Buarque de Hollanda en Río de Janeiro, sólo hay cursos particulares dentro de programas de letras, antropología, sociología y comunicología. Pero la diferencia entre EE.UU. y América Latina es todavía mayor. Cada vez más los ECLA forman parte de la alianza entre Area y Ethnic Studies, resolviendo así la tensión entre lo latinoamericano y lo latino, a menudo sin examinar las diferencias. Esto se debe a su función dentro de la academia estadounidense: los ECLA, sobre todo en el ámbito de grado, satisfacen requisitos curriculares de “culturas mundiales”, es decir, culturas que se suponen no herederas de occidente. Puesto que el multiculturalismo responde a políticas gubernamentales y mercadológicas (sobre todo en la publicidad, los medios y el consumismo) no debe sorprender que haya un nutrido nicho para la oferta latinolatinoamericana.

Esta institucionalidad de los ECLA en EE.UU. conduce a un fenómeno notable: que en EE.UU. se diseñen los ECLA continentales mientras que en América Latina lo que se suele conocer y estudiar son los EC nacionales. Esta diferencia cobra mayor peso al considerar la crisis económica por la que está pasando América Latina, teniendo en cuenta que las políticas de reajuste han reducido el alcance de las universidades latinoamericanas. No hay manera que se pueda competir y los profesores residentes en EE.UU. han logrado

\footnotetext{
${ }^{1}$ Los "estudios de área" son en su mayor parte creación de la academia estadounidense, sobre todo a partir de la revolución cubana en los 1960 (si bien ya existían desde los años 1930), para conocer y así mejor negociar las relaciones externas en América Latina, la URSS, África y Asia. Cada una de estas áreas de producción de conocimiento correspondía a los intereses estratégicos estadounidenses. Los "estudios étnicos" surgen a partir de las políticas de derechos civiles para minorías racializadas a fines de los 1960, cuando se forman programas de estudios negros, chicanos y puertorriqueños. Con el final de la guerra fría en los 1990 y con el debilitamiento de las movilizaciones minoritarias, ambos tipos de estudios perdieron importancia en la sociedad estadounidense. No obstante, se pensó (con el apoyo de fundaciones como la Ford, que había invertido \$270 millones en los estudios de área y que no quería verlos morir) que podrían reanimarse ambas iniciativas, sobre todo aliándolas o fundiéndolas. Véase Yúdice, en prensa.
} 
una importancia inusitada en América Latina que invierte la situación de hace 30 o aun 20 años, cuando los escritores e intelectuales latinoamericanos eran muy solicitados en EE.UU. Si hace 20 años un Ángel Rama o una Beatriz Sarlo impartían sus conocimientos específicamente latinoamericanos, hoy en día los que se han formado según corrientes intelectuales en EE.UU., como Walter Mignolo o John Beverley, desempeñan esa función en el sur. ${ }^{2}$ Podría decirse que esta situación refleja el transnacionalismo característico del fin del siglo pasado y comienzos del actual, pero transnacionalismo e igualdad de condiciones no es lo mismo. Los ECLA deben reflexionar sobre esta desigualdad y hasta aspirar a modificarla, acaso estableciendo alianzas entre universidades del norte y las del sur, sin que las primeras dictaminen las condiciones de esas alianzas ni desencadenen una fuga de cerebros trayéndose a los mejores estudiantes y profesores a EE.UU.

A pesar de estas diferencias, creo que hay un interés compartido por todos los que participan en los ECLA, que es, como viene enfatizando con ahínco Daniel Mato desde hace años, la relación entre las manifestaciones culturales (artísticas, mediáticas o comunitarias) y las relaciones de poder (clasistas, raciales, empresariales, institucionales y geopolíticas). En el encuentro de LASA se debatieron al menos tres posiciones respecto a esta relación: (1) que los ECLA institucionalizados contribuirán a formar una conciencia progresista en los egresados que salgan a trabajar en otros campos (Román de la Campa); (2) que la institucionalización misma y la búsqueda de una efectividad social o política (v.gr., que los ECLA conduzcan a un cambio, revolucionario para algunos y reformista para otros) necesariamente someten el pensamiento y la cultura a criterios funcionalistas (Alberto Moreiras, Nelly Richard); (3) que no existe un afuera del entorno institucional y por lo tanto el efecto que los ECLA puedan tener siempre es algo negociado, gestionado y administrado (George Yúdice).

En el LASA del 2001 no surgió el debate entre latinoamericanos y latinoamericanistas residentes en EE.UU. como en el LASA de la primavera de 1997. En su ponencia magistral, Hugo Achugar hizo una crítica implícita a John Beverley por importar los estudios subalternos de la India y Sud Asia al contexto latinoamericano, y a Walter Mignolo por tomar como paradigma para el estudio de lo latinoamericano el pensamiento fronterizo o border thinking que éste derivaba del trabajo de Gloria Anzaldúa y otros intelectuales chicanos. Lo que suscitó la crítica de Achugar, y también la de Nelly Richard en otro panel, fue el sometimiento de lo latinoamericano como objeto de estudio a paradigmas teóricos que, se decía en ese entonces, no surgían de las realidades locales. Este debate se diseminó en las páginas de la Revista de Crítica Cultural con las respuestas de Mabel Moraña (1997) y Federico Galende (1996), foro en el que ya había salido la

\footnotetext{
${ }^{2} \mathrm{Al}$ decir que se han formado en EE.UU., no me refiero sólo al lugar en el que obtuvieron su Ph.D., sino más bien al lugar de cuyas corrientes intelectuales-académicas derivaron su orientación crítica. Este es el caso de Mignolo, que si bien se doctoró en semiótica en París no obstante desarrolló su interés por la epistemología fronteriza en relación con los estudios minoritarios estadounidenses, sobre todo de los intelectuales chicanos, así como su elaboración del poscolonialismo y del subalternismo a partir del trabajo de intelectuales sud-asiáticos residentes en EE.UU. Desde luego, no argumento que la rica elaboración crítica de Mignolo se limite a estos aportes, sino que condicionan su discurso y contornan su lugar de enunciación.
} 
crítica contundente de Beatriz Sarlo a los estudios culturales. Como si se tratara de una plaga, Sarlo preguntó: “¿Cómo se mantiene una sociedad después del multiculturalismo?”. En “El boom del subalterno”, Moraña criticó los criterios poscoloniales, subalternistas, minoritarios y sexuales a partir de paradigmas prevalecientes en EE.UU. Además, se juzgaba que los estudios culturales habían abandonado la literatura.

3. LOS ESTUdiOS CULTURALES LATINOAMERICANOS EN TIEMPOS DE TERRORISMO, VIGILANCIA Y GUERRA MUNDIAL

Pero estos debates, por importantes que sean, quedan en un segundo plano al reflexionar sobre las repercusiones del atentado a las torres gemelas y la llamada "nueva guerra” del gobierno de Bush contra Afganistán y las redes de terroristas que se encuentran en todas partes del globo. Parecería no tener nada que ver con América Latina ni con los ECLA, pero considérense las siguientes repercusiones: de inmediato se cierran las fronteras entre EE.UU. y México que el gobierno de Fox estaba tratando de abrir aún más; el Plan Colombia, que debe eliminar el problema del narcotráfico y de la guerrilla, se asimila al nada nuevo "nuevo orden mundial” de vigilancia y guerra a terroristas; la piratería de los productos de la industria global del entretenimiento y de la industria digital también se asimilan a las redes terroristas y se intensifica el control de lugares como Ciudad del Este o Manaos; los sistemas de televisión, desde donde la mayoría de los residentes de América Latina recibe sus informaciones, no tienen acceso a otras informaciones sobre la guerra que las que permita el gobierno estadounidense que impone una censura que el Big Brother de Orwell o Huxley envidiaría. Todo esto quiere decir que la ayuda económica, que ha servido para salvar a los inversores en tiempos de crisis económica, ya ni tendrá que legitimarse mediante políticas para mitigar la pobreza y fortalecer la sociedad civil. Pero aún más, ya ni se aprobarán los bailouts para países cuyas economías ya no forman parte significante del nuevo régimen de acumulación; de ahí la negación de préstamos para salvar la economía argentina. La seguridad del orden mundial ya no se medirá en esos términos, sino que irá a producir todavía más ganancias para las tres industrias que apoya Bush: el complejo militar-industrial, la industria farmacéutica (que por lo visto ya es parte del complejo militar) y el petróleo. Habría que añadir, además, lo que James Der Derian (2001) ha denominado la red "militar-industrial-mediática-y-deentretenimiento".

Hay que reorientar a los ECLA a una crítica del nuevo o acaso no tan nuevo imperialismo, con su alianza de intereses militares y empresariales, y como veremos, mediáticos. Esta crítica acaso posibilite que se mantenga la alianza entre académicos progresistas estadounidenses e intelectuales latinoamericanos, cuyo puente podría ser el movimiento de crítica a la globalización al estilo del Foro Económico Mundial.

\section{4. ¿¿RENACIMIENTO DE LA CRítICA AL IMPERIALISMO MÁS-QUE-CULTURAL?}

Tocaré todos estos puntos, pero primero cabe señalar que hay una nueva actitud hacia Estados Unidos en América Latina, cuyas poblaciones se preguntan, como en otras partes del mundo, si los atentados no serán la revancha que se merecen. En otras palabras, los 
atentados dieron lugar a numerosas expresiones de antiimperialismo, notando que EE.UU. se había mantenido más o menos a salvo a pesar de las innumerables intervenciones en el Medio Oriente y otras regiones. Ahora, finalmente, les tocaba experimentar la violencia que ha sido cotidiana en Israel, Palestina, Irak y otras partes del mundo. Considérese, por ejemplo, las opiniones de David Viñas, Hebe Pastor de Bonafini, Sergio Schoklender y Vicente Zito Lema, expresadas en una clase pública "Sobre la guerra imperialista” en la Universidad Popular de las Madres de la Plaza de Mayo. Viñas consideró los atentados como expresión de la lucha de clases, una revancha desde abajo contra "la violencia institucional del imperio", contra "la violencia enquistada arriba", y comparó las acciones de "los sometidos, los humillados del mundo" a las de Robespierre y Castelli. ${ }^{3}$ Zito Lema concordó con el “análisis” de la lucha de clases y caracterizó a Bin Laden como un revolucionario y lo comparó con San Martín, Belgrano, Artigas, el Che Guevara y sus “compañeros caídos en combate” durante la guerra fría. Para Shoklender se trataba no de actos terroristas sino, usando la misma terminología ofuscadora del gobierno estadounidense, de "operaciones quirúrgicas" contra "los centros precisos de poder” del "enemigo que nos está destruyendo”. Añadió que se alegró al constatar que los EE.UU. no eran invulnerables y que "tenemos la posibilidad de resistir y enfrentarlos”. Bonafini, quien se encontraba en Cuba durante los atentados, declaró su admiración por los que pilotearon los aviones, observando que "declararon la guerra con sus cuerpos, manejando un avión para estrellarse y hacer mierda al poder más grande del mundo. Y me puse contenta”. Vio los atentados, además, como el saldo de una deuda de los desaparecidos argentinos para con los estadounidenses, pues ahora viven el "miedo que nos metieron a nosotros, con la persecución, con la desaparición y con la tortura [...] Ese pueblo que se calló y aplaudió las guerras”.

Demás queda explicar que las víctimas de los atentados no se limitaron a los capitalistas. Las listas de los más de tres mil muertos revela que la mayoría eran trabajadores, muchos de ellos extranjeros de unos 80 países (entre ellos muchos latinoamericanos), para no hablar de las minorías racializadas en EE.UU., como los inmigrantes indocumentados, centenares de los cuales murieron en las torres (Robles 2001). Al menos otros 700 se quedaron sin trabajo (Moreno Gonzales 2001). Tampoco me parece acertado considerar a los autores de los atentados como revolucionarios "que dieron y entregaron sus vidas para un mundo mejor” (Bonafini), pues la lucha geopolítica en la cual participan — que hay que reconocer no fue iniciada por ellos sino por EE.UU. y otros países europeos - no es para establecer un mundo mejor en el sentido socialista, sino para consolidar regímenes autoritarios (como Irak), teocráticos (como Afganistán) y militares (como Paquistán), que no respetan derechos políticos, civiles o humanos.

Como explican Verbitsky y Rolando Astarita, quien renunció a su puesto en la misma Universidad de las Madres de la Plaza de Mayo tras escuchar las palabras de sus colegas, el billonario Bin Laden no representa la causa de los desheredados del mundo, ni los atentados son parte de la lucha de clases, cuyos parámetros son otros, ni se trata de acciones que promuevan el socialismo. Para Astarita el socialismo “jamás ha defendido la matanza

\footnotetext{
${ }^{3}$ Todas las citas a Viñas, Bonafini, Shoklender y Lema provienen del reportaje de Verbitsky (11 de octubre de 2001). Los textos completos se pueden consultar en "Resumen Latinoamericano".
} 
indiscriminada de personas” ni su objetivo es la "venganza” invocada por Bonafini sino “acaba[r] con la propiedad privada de los medios de producción” (Astarita 2001). Verbitsky añade que "Mientras [Viñas] no ... revele [sus fuentes de información respecto a que Bin Laden dirigió los atentados], parecería que sus autores no han sido proletarios en lucha por la revolución socialista, sino el régimen teocrático de Afganistán, supresor de todas las libertades que permitirían vivir y desarrollar su obra en ese país a los intelectuales críticos, ateos, socialistas y judíos como Viñas. Y a sus mujeres, detalle no menor".

Que los promotores de la justicia para los desaparecidos en la guerra sucia descarten los derechos humanos como campo de lucha es sintomático de un cambio preocupante en los países latinoamericanos. Zito Lema, por ejemplo, despreció a los intelectuales argentinos que "lloran por el poderoso cuando son cómplices cotidianos de la muerte" y que no "habl[an] de derechos humanos" cuando "35.600 niños [...] mueren todos los días”. Si bien es verdad que EE.UU. han usado la retórica de los derechos humanos hipócritamente, no para sancionar a las dictaduras que cuidan de sus intereses sino para intervenir en los países con cuyas políticas y acciones no están de acuerdo, también es importante tener en cuenta que sin la política de los derechos humanos no se habría logrado combatir a sus violadores y llevarlos a la justicia, como se ha hecho inclusive con figuras antes consideradas intocables como Pinochet.

¿Después de dos décadas de activismo de derechos humanos estamos viendo su crisis? Si bien es verdad que las guerras desatadas por EE.UU. para defender el libre flujo del capital son una forma del terrorismo, ¿ello justifica que algunos activistas de los derechos humanos aplaudan los ataques terroristas a estadounidenses, como si se tratara de un desquite por las desapariciones en el Cono Sur? ¿Será que se ha perdido el juicio que pueda distinguir entre la resistencia a la opresión y los asesinatos cometidos por grupos guerrilleros como las FARC? Verbitsky nos recuerda que los argumentos con que Trotsky se opuso al terrorismo individual en 1911 todavía son válidos para el terrorismo organizado por el Estado o por redes religiosas o políticas, pues cuanto más exitoso, tanta menos “autoorganización y autoeducación” protagonizarán las masas y tanto mayor y salvaje será la represión policial. De hecho, como ya observé más arriba, los atentados le han dado a EE.UU. la “justificación” para extender sus aparatos de espionaje, vigilancia y control militar a lo largo del mundo, lo cual se verifica con el entusiasta apoyo del Reino Unido, Australia y el endiosamiento de la OTAN y las alianzas con los gobiernos de occidente, alianzas que restringirán los derechos políticos y humanos. Ya el Secretario de Justicia Aschcroft identificó a 36 grupos que serán perseguidos, como también a cualquiera que tenga relaciones con ellos. Esto produce enormes peligros para el incipiente movimiento antiglobalización, cuyas acciones ahora serán mayor objeto de represión que en Seattle o Génova. Ya han sido detenidas miles de personas por su parecido físico o por sospechas suscitadas por los libros que leen, como verifica la detención de Tariq Ali, que llevaba un libro de Marx cuando estaba por abordar un avión en Alemania (Ali 2001). 


\section{VigILANCIA OMNÍMODA}

Las acciones de los terroristas y del gobierno de Bush se alimentan recíprocamente, pues por un lado la guerra desatada contra Afganistán fomenta la solidaridad de muchos musulmanes que se opondrían a la represión de libertades políticas, civiles y culturales por parte de los estados teocráticos, y por otro “justifica” la mayor penetración del militarismo y de las fuerzas y tecnologías de seguridad estadounidenses a lo largo del mundo. Así se logra además incorporar a su radio de acción luchas que poco tienen que ver con el conflicto en el Medio Oriente y en Sud Asia, como es el caso de los narcotraficantes y las guerrillas en Colombia. Si bien las FARC parecen haber perdido toda consecuencia ideológica con los secuestros y asesinatos ya no sólo a los dirigentes de la contrainsurgencia sino también a civiles y hasta a misioneros indígenas de izquierda, ello no quiere decir que haya un parentesco entre ellos y las fuerzas del Al-Qaeda, como ya han declarado representantes de EE.UU. Por ejemplo, Anne Patterson, embajadora estadounidense en Colombia, alegó que “las FARC y Bin Laden tienen la misma hipocresía moral y falta de ideas. Los talibanes afganos no representan al Islam y los guerrilleros colombianos no buscan la justicia social” (citada en Kollmann).

La ayuda militar a Colombia ya se “justificaba” por el combate al narcotráfico, pero como señalan analistas de esa situación, la acción estadounidense en Colombia también responde a los llamados de las empresas estadounidenses, pues la guerra civil no facilita mantener sus ganancias. A pesar de la gran pérdida de vida de civiles y la violación de derechos humanos por los guerrilleros, los narcotraficantes, las fuerzas armadas y los paramilitares, las empresas han logrado que los \$1,6 mil millones en ayuda militar estadounidense se usen para defender sus propios intereses (Staples). Y como si esto no fuera suficiente, la propaganda estadounidense ya está estableciendo los vínculos entre Bin Laden, las FARC, el narcotráfico (heroína y cocaína) y la Triple Frontera entre Argentina, Brasil y Paraguay. Habiendo perdido toda legitimidad, los militares latinoamericanos procuran asociarse a las reforzadas políticas de seguridad de EE.UU. (v.gr., Operación Centauro) para aumentar sus presupuestos en una época en que los gobiernos latinoamericanos han tenido que apretar los cinturones (Bonasso). ${ }^{4}$ Más problemática todavía es la inclusión en las listas de terroristas del gobierno de Bush de grupos progresistas latinoamericanos, sobre todo insurgencias antiautoritarias o para expandir los derechos de minorías, como es el caso de los zapatistas. El comandante del United States Southern Command (USSOUTHCOM) en América Latina ha dicho que su propósito es eliminar el tráfico de armas y drogas (Pace), pero los efectos de esa vigilancia incluye la represión de la oposición popular al neoliberalismo, acaso el verdadero blanco de esas políticas militares. Las protestas que echaron abajo al gobierno argentino en

\footnotetext{
4. La Operación Centauro fue desarrollada por la CIA y el Secretariado de Inteligencia de Argentina (SIDE) para vigilar la triple frontera entre Argentina, Paraguay y Brasil luego del atentado a la Asociación Mutual Israelí-Argentina (AMIA). El propósito era infiltrar a grupos islámicos fundamentalistas, como la Hezbollah, que según la inteligencia argentina, tenía planes para poner bombas en la embajada estadounidense en Asunción (Bonasso 2001).
} 
diciembre y enero de 2002, son un ejemplo de la inestabilidad que preocupa a los agentes de esta vigilancia.

¿Por qué estas conexiones? Según el reportaje de Kollman, la guerra en Afganistán ha suscitado el traslado a Colombia de la producción de heroína, droga que tiene diez y siete veces el valor de la cocaína. EE.UU., además, teme que el conflicto colombiano migre hacia el vecino productor petrolero Venezuela, cuyo presidente Hugo Chávez ya ha declarado en más de una ocasión su solidaridad con la guerrilla. Las conexiones que EE.UU. viene trazando, pues, son parte de una estrategia de fortalecimiento de las redes de vigilancia y militarización en distintas regiones, en este caso, haciendo entrar a Argentina y Brasil en el conflicto colombiano, para pactar el desarme, como ocurrió con el IRA irlandés, o para intervenir en la "acción armada contra los revolucionarios colombianos, a los que ellos llaman — como en Afganistán—la narcoguerrilla” (Kollmann).

\section{SEguridad PARA El RÉgimen de ACUMUlación}

Si es verdad, como sugiere Staples, que los atentados han dado un nuevo impulso a las fuerzas políticas y económicas que vienen generando la globalización de manera que estamos entrando en un nuevo período histórico, vale la pena considerar cómo esto afecta a América Latina. Pero primero es necesario entender el contexto global en que se inserta el caso latinoamericano. Creo que el nuevo régimen de vigilancia y seguridad amenaza sobremanera a los movimientos sociales y de sociedad civil, neutralizándolos represivamente o absorbiéndolos a la maquinaria de la seguridad, que toma varias formas sociales y culturales. De ahí la importancia de las políticas culturales en este nuevo período. La globalización generada por la transnacionalización de las empresas y los flujos financieros ha producido mayor desigualdad que en los veinticinco años después de la segunda guerra mundial. Pero en lugar de diseñar políticas para contrarrestar la pobreza generada por la globalización, las instituciones financieras y comerciales internacionales (Fondo Monetario Internacional, Banco Mundial, Organización Mundial de Comercio, etc.) se limitan a crear nuevos programas para rebajar o eliminar la enorme deuda externa de los países más pobres, a la vez que recurren crecientemente a la cultura como panacea (Wolfensohn; World Bank). Pero aun cuando se elimine la deuda externa de los países pobres, eso no afecta la estructura que genera la desigualdad, que es la legalización de la división internacional del trabajo y la producción de propiedad.

Las privatizaciones llevadas a cabo en casi todos los países latinoamericanos son un ejemplo de la transformación del bien patrimonial en bien privado en el transcurso del Estado-Nación moderno al estado neoliberal. Herscovici explica que la designación de patrimonio o propiedad privada a ser valorada conforme al mercado no responde a factores naturales sino más bien a decisiones políticas que corresponden a la inserción en la economía mundial (Herscovici 55). El control que los países centrales ejercen sobre los otros se ve claramente en las políticas de patentes y propiedad intelectual que regulan la producción internacional de medicamentos. Brasil, la India y Sudáfrica llevan años tratando de conseguir cierta autonomía respecto del régimen de propiedad intelectual que les ha prevenido de producir medicinas para combatir el SIDA a precios que posibiliten 
que sus ciudadanos puedan sobrevivir la epidemia (CPTECH 2001). La industria farmacéutica ha combatido con bastante éxito, si bien ha habido casos recientes en los que estos países lograron una rebaja en el pago de los derechos tras amenazar con producir versiones genéricas. En el Brasil, por ejemplo, existe una ley que dictamina que se pueden producir versiones genéricas en casos de emergencia. Ante este caso, es interesante observar la política del gobierno estadounidense que se resistió a comprar versiones genéricas de los antibióticos contra el anthrax, mientras que Canadá autorizó la producción genérica.

Las desigualdades generadas por estos regímenes de derechos de propiedad intelectual, pues, agudizan la pobreza de otra manera. Puesto que todas las mayores empresas del mundo son conglomerados globales con sede en los países del Grupo de los Siete (G-7), no se ha permitido que los países metropolitanos periféricos (Europa del sur, Europa oriental) y mucho menos los países en vías de desarrollo, inclusive con economías del tamaño de la brasileña, la novena del mundo, puedan aprovecharse de los instrumentos de acumulación. Las universidades estadounidenses y europeas, por ejemplo, que en alianza con las empresas son motores de innovación para la acumulación, no tienen contrapartes en América Latina. De hecho, se está destruyendo el modelo de la universidad pública, inclusive las más importantes como UNAM, USP y UBA, y se está fomentando la creación de universidades privadas donde se llevará a cabo la Investigación y Desarrollo (I\&D) bajo contratación para los conglomerados globales. Esta transformación tiene la desventaja, además, de que se cerrará la vía de acceso de las capas sociales ya no sólo más bajas sino también de muchas capas medias al ascenso social.

Según el Ministro de Educación del Brasil, Paulo Renato de Souza, “a ênfase no ensino universitário foi característica de um modelo de desenvolvimento auto-sustentado que demandava criar pesquisa e tecnologias próprias [...] hoje este modelo está em agonia terminal” (Souza). Según Souza, el entrelazamiento internacional de la globalización hace imposible esta autonomía; por lo tanto el ministerio apoya "o acesso ao conhecimento fica facilitado, as associações e joint ventures se encarregam de prover as empresas de países como o Brasil do know-how de que necessitam”. Añade que la terciarización de las universidades, como en Corea, "faz mais sentido do ponto de vista econômico". Una crítica de estas políticas gubernamentales, “Em defesa da Universidade Pública”, señala que puesto que el $99 \%$ de las patentes pertenecen a las empresas multinacionales del G7, el alto costo de los derechos de propiedad intelectual impide el acceso de las mayorías a medicamentos, productos agrícolas, etc. (ANDES-SN 2001).

El debilitamiento del sistema universitario, y por ende de su posible función como generador de propiedad intelectual, ya se ha realizado con los cortes presupuestarios para las noventa y nueve universidades federales, que no han recibido aumento desde 1995. Añádase que el presupuesto para bibliotecas y servicios relacionados ha caído $80 \%$. La situación es todavía peor en la Argentina donde el nuevo acuerdo con el FMI, inspirado en las violentas medidas adoptadas en el país, ya produjo cortes más violentos como la reducción de salarios, por medio de la rebaja de gratificaciones y otras medidas que el Ministerio de Educación presentó al Congreso Nacional, augurando una mayor transición a la privatización de la universidad, aboliendo la estabilidad de empleo, lo cual hace 
imposible que el profesorado pueda planear sus programas con autonomía. Es justamente esta autonomía la que no interesa al capital (ANDES-SN 2001).

Como señaló Verbitsky, los atentados en EE.UU. no han "destruido el poder más grande del mundo”, declaración de Bonafini que le parece “de una patética ingenuidad”, pues el " software que mide el riesgo país ya fue instalado en otro edificio”. Es decir, las tecnologías de control operan como se dice en computación, en el fondo o background, a escondidas, sin que uno se dé cuenta. No se dispone de suficiente perspectiva histórica para determinar cómo será el futuro del régimen de acumulación actual ni tampoco de la actual guerra para sostenerlo ante los contraataques terroristas. Lo que sí parece evidente es que se está gestando una nueva manera de controlar las contingencias en un mundo en el que las nuevas tecnologías las han multiplicado exponencialmente. Este nuevo régimen de control es sumamente cultural, aunque no lo parezca. No se trata tanto del tipo de imperialismo cultural impugnado por Dorfman y Mattelart hace tres décadas, si bien, como nos dice Benjamin Barber (1995), el Jihad se ha levantado contra McMundo. Esta interpretación es la más civilizacional, en el sentido que la esboza Samuel Huntington (1993) en su notorio ensayo "The Clash of Civilizations”. Pero más que el consumismo en sí, que erróneamente se supone repudiado en el Medio Oriente, se trata del régimen de propiedad y acumulación que lo subyace.

Considérese que el mundo empresarial globalizado requiere de las fuerzas de seguridad para proteger sus ganancias. Esto es evidente en el caso de la industria del entretenimiento (cine, TV, música, libros, videojuegos) y de otros productos digitales, que la piratería o "tráfico de productos de entretenimiento” hostiga, expoliando una cantidad equivalente a la que esa industria genera globalmente. Para tener una idea de la enormidad de esa "expoliación”, considérese que en 1998 el sector de artes, entretenimiento, comunicaciones y copyright alcanzó unos \$360 mil millones sólo en los EE.UU. (Arthurs y Hodsoll 104). Como dice el columnista del New York Times Thomas Friedman, "la mano oculta del mercado siempre opera con un puño oculto. McDonalds no prospera sin McDonnell Douglas, fabricador de jets de caza F15. Y el puño oculto que mantiene al mundo seguro para las tecnologías del Silicon Valley no es sino el Ejército, la Marina y la Fuerza Aérea de EE.UU.” (citado en Staples).

\section{El CONTROL COMUNiCACiOnAL}

Los sistemas de comunicación también participan de ese control mundial. La transformación de los sistemas de comunicación mediante procesos neoliberales (privatización, desregulación, eliminación de los servicios proporcionados por el estado benefactor por razones políticas y económicas) resulta en una recomposición y resignificación de territorios y públicos. La transnacionalización y (neo)liberalización de las industrias culturales impone (1) la necesidad de insertarse a una economía supranacional y (2) reestructuraciones para facilitar esa inserción que responde a "una dialéctica de la uniformización y de la diferenciación” (Herscovici 58). Por una parte, se uniformizan protocolos jurídicos, tecnologías y formas administrativas; por otra, la acomodación al mercado transnacional requiere la generación de diferencias locales que posibiliten la 
rentabilidad de contenidos allende las fronteras territoriales. “[C]ada espacio geográfico precisa diferenciarse y construir su imagen mediática a fin de valorarse con relación al exterior para poder insertarse en esas redes internacionales; la cultura es ampliamente utilizada en la construcción de esta imagen mediática” (Herscovici 58-9). El hecho de que la valorización de localidades y de sus contenidos se hace a partir de la generación de esas diferencias (orquestadas en un principio por el entorno mercadológico transnacional) obliga a que los procesos de formación de identidades culturales obedezcan a mandatos performativos. En otras palabras, las diferencias no se pueden pensar fuera del entorno del cual derivan su valor; de ahí que se pueda argumentar que las diferencias se constituyen dentro de los procesos de globalización (Lacarrieu 4-5).

Tomar conciencia de esto complica nuestra comprensión de los programas de reivindicación de lo local, pues nos damos cuenta que la diferencia es el recurso que permite la valoración, inclusive en iniciativas de la sociedad civil que no corresponden directamente al mercado. Los territorios que no puedan o no respondan al imperativo performativo proyectado desde el campo de fuerzas serán excluidos de o marginados en los procesos de producción de valor. Desde luego, me refiero a tendencias de absorción de esos movimientos que como los sem terra en el Brasil o los neozapatistas en Chiapas procuran participar en la distribución de bienes y servicios, sean estos del Estado, del mercado o de la sociedad civil. Pero también hay movimientos de resistencia, por ejemplo las Madres de la Plaza de Mayo, o Hijos en el contexto argentino, que procuran rescatar la memoria de lo que el Estado y el mercado han invisibilizado y que la llamada sociedad civil no facilita siguiendo las políticas de representación vigentes.

Los medios cada vez más colaboran en el sistema de control. Como constataron varios teóricos de las nuevas formas de guerra reticular (Arquilla y Ronfeldt, Stephanson, Castells), quien controla la información y su encuadramiento gana la guerra (Garreau). De ahí la importancia de una nueva censura que se vislumbró durante la Guerra del Golfo Pérsico. El estado militarizado estadounidense ya no permitió que los periodistas buscaran información por cuenta propia, sino que los encaminaron a los lugares y escenarios que el gobierno quería que se vieran. Esta vez, el gobierno ha sido todavía más descarado (acaso más desenmascarado). El 10 de octubre Condoleezza Rice, consejera de Bush para seguridad nacional, les pidió a las redes televisivas que no mostraran sin editar mensajes enviados por Osama Bin Laden. Todas las redes accedieron (Roberts). Más aún, como sabemos, la televisión se ha convertido en un arma de la guerra del gobierno. Pero esta guerra no se limita al espacio nacional sino que, debido a la influencia de los conglomerados mediáticos y de entretenimiento, se ha presionado para que la televisión en América Latina también edite sus mensajes. Los televidentes de CNN en América Latina se quejaron de las evidentes estrategias de manipulación. Observa Piscitelli (2001) que “CNN violó sus propios manuales de estilo que tenían hasta hace poco, como ejes rectores la religión del uso del sonido ambiente, y la inexistencia de trucos de edición, así como la obligatoriedad de la diseminación de opiniones alternativas. En los tres aspectos CNN rompió sus códigos arrastrada obviamente por la ola de patriotismo y por la sed de venganza”.

En Estados Unidos, todas las redes televisivas aportaron cobertura hasta la náusea sin pasar anuncios. La razón por la cual hubo esta pérdida de ingresos publicitarios no se 
debió sólo al patriotismo y la sed de venganza, sino al galardón que les daría el gobierno. Sólo dos días después del atentado, la Federal Comunications Commission (FCC) eliminó los últimos artículos de regulación que quedaban después de dos décadas de desregulación neoliberal (FAIR 2001). Así se logró que los dueños de periódicos también puedan comprar canales de televisión y operar en la misma área nacional. Este tipo de convergencia ya existe en América Latina. Con ello se logra que los conglomerados gigantescos como News Corporation (de Rupert Murdoch), AOL — Time Warner (dueño de CNN), VIACOM y Disney, diseminen todavía más su influencia. Lo mismo acontece con la Rede Globo en Brasil y el Grupo Clarín en Argentina.

Y este acceso no se limita a la televisión. De hecho, el director de AOL confesó que su división de Internet ya colabora con el servicio de inteligencia, dándole acceso a la CIA al correo electrónico de sus suscriptores. Añade que no se opondrá a otras medidas antiterroristas solicitadas por el Departamento de Justicia (Roberts). Como dice Castells (1996), hay que combatir las redes con las redes (citado en Garreau), y no hay mejor vehículo para hacerlo que Internet. Esto implica la colección clandestina de información mediante tecnologías como los cookies. Por ejemplo, cuando el usuario navega la web para comprar boletos, los cookies se comunican con su computadora e instalan un software de vigilancia en ella que envía información cada vez que se usa. Los teóricos de la guerra reticular (netwar) describen una nueva estrategia según la cual se infiltran sitios de internet visitados por activistas y se les insertan software inteligentes que monitorean todas sus actividades, dando la impresión que estuvieran en el mundo del Truman Show (Arquilla y Ronfeldt).

\section{LA PRESCRIPCIÓN DE NARRATIVAS}

Hay pues que reconstruir nuestra comprensión de la totalidad, generar mapas cognitivos, como dijera Jameson hace dos décadas, pero no es evidente en qué registro se pueda lograr. Acaso se trate de adoptar la forma de redes, que no operan sólo en relación con una localidad fija, sino en conexiones relativamente autónomas, como las que ya adoptó el capitalismo, según Manuel Castells (2001), y que por lo visto también adoptaron los terroristas y el movimiento antiglobalización. Esta forma de organización y de monitoreo fomenta nuevas experiencias culturales, inclusive virtuales, pues muchas de ellas están u ocultas o son generadoras de verosimilitud. Lo más insidioso acaso sea que nos están preescribiendo las narrativas.

En 1996, Arquilla y Ronfeldt escribieron The Advent of Netwar para el Instituto Nacional de Investigación en Defensa, un centro de investigación y desarrollo financiado por la Oficina del Secretario de Defensa, los Jefes del Estado Mayor y otras agencias de defensa. Arquilla y Ronfeldt pronosticaron que los que posean mejor inteligencia ganarán la guerra entre redes humanas. También hicieron algunas recomendaciones tácticas para combatir las redes humanas con contra-redes que ya habían dado resultado contra los hackers. Estas recomendaciones incluyen: infiltrar las redes enemigas y hacer al más tonto creerse la figura más importante del núcleo; discernir a las figuras más peligrosas y cuestionar su lealtad al grupo; controlar las historias que la gente se cuenta al dar su razón 
de ser y de actuar. Según Ronfeldt, el relato terrorista "les da a sus seguidores una causa común - un nosotros contra ellos. Pareciera que en este momento EE.UU. tienen la ventaja a escala mundial en este respecto, pero podemos ver que los palestinos bailan en la calle [para festejar el colapso de las torres gemelas]. Según su relato, la maldad y la presencia estadounidenses son culpables de los problemas en el Medio Oriente. Nosotros tenemos que atacar esa parte del relato” (Arquilla y Ronfeldt).

Esta ingeniería narrativa se logra desprestigiando al enemigo; destruyendo sus redes de apoyo; infiltrándolo con grupos con valores diferentes, por tanto dividiéndolo y conquistándolo; aumentando el número de contra-redes humanas en la propia sociedad civil. En cuanto a los espectáculos, se opina que los ataques militares ya no proporcionan la única acción efectiva, pues cada vez que erran con sus misiles, muestran que no tienen información confiable y además hacen posible construir narrativas en nuestra contra (Stephenson, citada en Garreau).

\section{HOLLYWOOd DEFIENDE SUS INTERESES}

Para concluir, considero dos opciones respecto al lugar de la cultura en la nueva encrucijada. La primera es la que tomaron los militares al invitar a cineastas y guionistas como Steven E. De Souza y Joseph Zito, que dirigieron "Delta Force One”, para generar nuevos escenarios de terrorismo mediático (Roberts). También se usan las nuevas tecnologías de simulación hollywoodense para entrenar a los militares (Bart; Hart; "U.S. Army Goes Hollywood” 1999). Este uso de los medios sin duda apunta en direcciones muy distintas a los estudios mediáticos típicos de la academia estadounidense e inclusive a los de estudiosos como Martín-Barbero. Más aún, cuanto más participan los usuarios de estos escenarios preescritos, de estos parámetros para interpretar el mundo, tanto más control se ejerce en este nuevo régimen de vigilancia.

Detrás de la simulación, pues, se encuentra la violencia y el militarismo. Incumbe a los intelectuales y a los artistas combatir estos usos de los medios o, como en el caso del artista de medios estadounidense Jordan Crandall o de la ciberescritora Giselle Beiguelman, subvertirlos, opción que exploro en otro ensayo publicado recientemente en Revista Iberoamericana (Yúdice). Pero como dije al principio, me parece que acaso la cultura no sea el campo más apto para combatir el régimen de acumulación, que ya ha absorbido en gran parte la labor cultural. De hecho, Rifkin (2000) argumenta que la experiencia misma devino recurso de explotación en la nueva economía. Por tanto, se requieren prácticas que nos lleven más allá de las resistencias y subversiones, que a menudo no proponen nada nuevo o diferente.

Reflexionando sobre la bancarrota económica y política de la Argentina a comienzos de 2002, Gabriela Massuh se pregunta si “en medio de este marasmo de realidades urgentes [no sea posible] [...] detenerse en el único factor en el que la Argentina puede comenzar a rescatar el futuro. El único viaje al exterior para el cual no se necesitan divisas oficiales, paralelas o flotantes: la cultura” (Massuh). ¿A qué cultura se refiere? A la literatura, cine, música, pintura y otras artes que dan a Argentina una "visibilidad internacional [...] que acaso no se corresponda del todo con la realidad, pero es parte de nuestra intransferible 
y a garrotazos tallada identidad cultural”. Parece asomar en la perspectiva de Massuh el argumento de que si el Estado hubiese fomentado más, a la vez dando autonomía a estas expresiones, acaso habrían cundido nuevas formas de interacción en el país, ayudándolo a crear salidas ante el inminente desastre. A mi ver, la cultura podría ayudar a sobrevivir el desastre pero no a evitarlo. ${ }^{5}$ A estas alturas, los lugares comunes de los estudios culturales, sobre todo los de raigambre gramsciana, dejan mucho que desear. Estamos en una época distinta, en la cual se tiene que volver a dar prioridad a la economía política, inclusive a la economía política de la cultura.

\section{BibliografíA}

Ali, Tariq. “Germany’s Green Police State Busted in Munich”. 30 de octubre de 2001. http://www.counterpunch.org/wtcarchive.html

ANDES-SN (Seção Sindical dos Docentes da UFRJ). “Em defesa da Universidade Pública”. Boletim Especial. 20 de agostode 2001. http://www.adufrj.org.br/2001/ jornal200801/debate\%20010820.pdf

Arquilla, John y David Ronfeldt eds. Networks and Netwars: The Future of Terror, Crime, and Militancy. Rand, 2001. http://www.rand.org/publications/MR/MR1382/

Arthurs, Alberta and Frank Hodsoll. "The Importance of the Arts Sector: How It Relates to the Public Purpose.” Journal of Arts Management, Law, and Society 28/2 (1998): 102-8.

Astarita, Rolando. “Crítica al discurso de Hebe Bonafini y renuncia a la UPMPM”. 16 de octubre 2001.http://www.antroposmoderno.com/antro-articulo.php?id_articulo=12

Barber, Benjamin R. Jihad vs. McWorld: How Globalism and Tribalism Are Reshaping the World. New York: Times Books, 1995.

Bart, Peter. "Bush Crusade Goes Hollywood: Will He Bomb at Box Office?” Variety 7 (2001): http://dailynews.yahoo.com/htx/nm/20011107/en/industry-war_1.html

Beverley, John y José Oviedo eds. The Postmodernism Debate in Latin America. Boundary 2 20/3 (1993).

Bonasso, Miguel. "La CIA traslada a su agente local por una revelación de Página12” Página 12(2001): 14.http://www.pagina12.com.ar/2001/01-01/01-01-14/pag03.htm

Castells, Manuel. The Rise of the Network Society. Oxford: Blackwell, 1996.

The Internet Galaxy: Reflections on the Internet, Business, and Society. Oxford: Oxford University Press, 2001.

Castro-Gómez, Santiago y Eduardo Mendieta (Eds.). Teorías sin disciplina: Latinoamericanismo, poscolonialidad y globalización en debate. México-San Francisco: Porrúa-University of San Francisco, 1998.

CPTECH. "New Trade Agreement for the Americas Jeopardizes Brazil’s Acclaimed Generic AIDS Drug Program”. Remitido de prensa. 12 de abril de 2001. http:// www.cptech.org/ip/health/trade/FTAArelease04122001.html

\footnotetext{
${ }^{5}$ Massuh misma escribe: "En tiempos de escasez la cultura y los artistas son los que más saben de pobreza. Para alivio de todos sería imprescindible que ninguna subsidiariedad les recortase esas alas propias que con creces supieron conseguir en esta castigadora tierra”.
} 
Der Derian, James. Virtuous War: Mapping the Military-Industrial-Media-Entertainment Network. Boulder: Westview Press, 2001.

FAIR (Fairness \& Accuracy In Reporting). "FCC Moves to Lift Cross-Ownership Ban ”. 26 de octubre de 2001. http://www.fair.org/activism/ownership-comment.html

Galende, Federico. "Un desmemoriado espíritu de época. Tribulaciones y desdichas en torno a los Estudios Culturales (una réplica a John Beverley)”. Revista de Crítica Cultural 13 (1996): 52-5.

Garreau, Joel. “Disconnect the Dots”. 17 de septiembre de 2001. http://www.washtech.com/ news/regulation/12516-1.html

Hart, Hugh. "Bringing Hollywood Pizazz to Military Training”. The New York Times. 15 de noviembre de 2001. http://college4.nytimes.com/guests/articles/2001/11/15/ 884642.xml

Herscovici, Alain. “Globalización, sistema de redes y estructuración del espacio: un análisis económico”. Globalización y monopolios en la comunicación en América Latina. Hacia una economía política de la comunicación. Guillermo Mastrini y César Bolaño eds. Buenos Aires: Editorial Biblos, 1999. 49-60.

Huntington, Samuel P. “The Clash of Civilizations”. Foreign Affairs 72/3 (Summer, 1993): 22-49.

Kollman, Raúl. "Al-Qaeda en la triple frontera según la CIA: Argentina y el Plan Colombia”. Página 12. 30 de octubre de 2001. http://www.pagina12.com.ar/2001/ 01-10/01-10-30/pag04.htm

Lacarrieu, Mónica. “Construcción de imaginarios locales e identidades culturales en la mundialización”. Ponencia presentada en el Seminario "Nuevos retos y estrategias de las políticas culturales frente a la globalización”. Instituto d’Estudis Catalans, Barcelona 22 a 25 de noviembre de 2000.

Massuh, Gabriela. “¿Qué hacer con la cultura?” La Nación. 25 de enero de 2002. http:/ /www.lanacion.com.ar/02/01/25/do_369179.asp

Mato, Daniel ed. Prácticas intelectuales latinoamericanas en cultura y poder I. Revista latinoamericana de Estudios Avanzados -RELEA 14 (en prensa).

(Ed.). Prácticas intelectuales latinoamericanas en cultura y poder II. Revista Venezolana de Economía y Ciencias Sociales 7/3 (2001).

Moraña, Mabel. “El boom del subalterno”. Revista de Crítica Cultural 15 (1997): 48-53.

Moreiras, Alberto. "The Order of Order: On the Reluctant Culturalism of Anti-Subalternist Critiques”. Journal of Latin American Cultural Studies 8/1 (1999): 125-46.

Moreno Gonzales, John. "Mexico President Pledges Assistance, Vows to aid fight, Mexican families”. Newsday 5 de octubre de 2001. http://www.newsday.com/news/ local/newyork/ny-nymexvr2399512oct05.story

Pace, General Peter. “Testimony of Gen. Peter Pace, commander-in-chief, U.S. Southern Command, Senate Armed Services Committee”. The Center for International Policy’s Colombia Project. 27 de marzo 2001. http://www.ciponline.org/colombia/032701.htm Piscitelli, Alejandro. Editorial. Interlink Headline News, No. 2416. 11 de septiembre de 2001. http://www.ilhn.com/ediciones/2416.html

“Resumen Latinoamericano”. resumen@nodo50.org 
Robles, Frances. "Families afraid to Talk about Missing Relatives”. Miami Herald. 25 de septiembre de 2001.http://www.miami.com/herald/special/news/worldtrade/digdocs/ 046429.htm

Souza, Paulo Renato. Entrevista. Exame.10 de junio de 1996.

Staples, Stephen. “Global Cops: The Corporate Security State’s Assault on Democracy”. Trabajo presentado en la conferencia "Global Cops: The Corporate Security State's Assault on Democracy”, St. Joseph's Parish, Ottawa, CA. 5 de octubre de 2001.

“U.S. Army goes Hollywood for high-tech training”. CNN.com. 18 de agosto de 1999. http://www.cnn.com/US/9908/18/army.hollywood/

Wolfensohn, James D. (1998) "Culture and Sustainable Development: Investing in the Promise of Societies". Comentarios sobre la conferencia "Understanding Culture in Sustainable Development: Investing in Cultural and Natural Endowments". Washington, D.C.: World Bank y UNESCO. http://wbln0018.worldbank.org/ Institutional/SPRConferences.nsf/547251faccc07412852566b000715b0b/ c13d72362c137a95852566b2005baccd?OpenDocument\&ExpandSection=2\#_Section2

World Bank. Culture Counts: Financing, Resources, and the Economics of Culture in Sustainable Development. Memorias de la conferencia. Washington DC, 1999. h t tp: / / W B L N 0018 . Worldbank. org/Networks / E S S D i c db.nsf/ D4856F112E805DF4852566C9007C27A6/4D4D56F007815BD1852568C8006741DF Culture and Sustainable Development: A Framework Action. Washington DC, 1999.http://lnweb18.worldbank.org/essd/essd.nsf/9b1cfc683a76b671852567cb0076a25e/ fa8a463ac24a48668525684600720ce7 Documento abierto al público.

Yúdice, George. "Rethinking Area and Ethnic Studies in the Context of Economic and Political Restructuring”. Rethinking Area and Ethnic Studies. Juan Poblete, ed. Minneapolis: University of Minnesota Press, (en prensa). 